\title{
An Algebra for Integration and Analysis of Ponder2 Policies
}

\author{
Hang Zhao \\ Department of Computer Science \\ Columbia University \\ zhao@cs.columbia.edu
}

\author{
Jorge Lobo \\ IBM T. J. Watson Research Center \\ USA \\ jlobo@us.ibm.com
}

\author{
Steven M. Bellovin \\ Department of Computer Science \\ Columbia University \\ smb@cs.columbia.edu
}

\begin{abstract}
Traditional policies often focus on access control requirement and there have been several proposals to define access control policy algebras to handle compositions and interactions. Recently, obligations are increasingly being expressed as part of security policies. However, their compositions and interactions have not yet been studied adequately. In this paper, we propose a policy algebra capturing both access control and obligation policies. The algebra consists of two policy constants and six basic operations. It provides language independent mechanisms to manage policies. As a concrete example, we instantiate the algebra for the Ponder2 policy language.
\end{abstract}

\section{INTRODUCTION}

Maintaining security policies in dynamic environments such as mobile networks or virtual environments where devices in the network or entities in the virtual organization share resources requires mechanisms to compose and integrate security policies. Prior to this work, we have proposed an algebra for firewall policy integration [10]. It will be interesting to see how the algebra could cope with high level policy languages. An algebra for fine-grained integration of XACML policies is introduced in [8]. XACML [1] is the OASIS standard language for specification of access control policies. Their algebra consists of four basic operators and two policy constants and is able to support the specification of a wide range of integration constraints.

Traditional security policies largely focus on the specification and management of access control requirement [3], [9], however the availability of services in many applications often further requires obligation requirements (see for example in [5]). The questions of how to understand the interactions between access control policies and obligation polices, and how to integrate and compose policies to enforce consistency in a policy-based system, have not yet been adequately investigated. Therefore, in this paper, we further extend the algebra defined in [8] by introducing obligation requirement to support the integration and analysis of Ponder 2 policies.

Research was sponsored by the U.S. Army Research Laboratory and the U.K. Ministry of Defence and was accomplished under Agreement Number W911NF-06-3-0001. The views and conclusions contained in this document are those of the author(s) and should not be interpreted as representing the official policies, either expressed or implied, of the U.S. Army Research Laboratory, the U.S. Government, the U.K. Ministry of Defence or the U.K. Government. The U.S. and U.K. Governments are authorized to reproduce and distribute reprints for Government purposes notwithstanding any copyright notation hereon.
The rest of the paper is organized as follows. SECTION II introduces background knowledge concerning the algebra for XACML policies and the Ponder2 language. SECTION III presents the detailed policy algebra, discusses its properties and expressiveness. Mechanisms for policy integration and analysis are described in Section IV. Finally, Section V concludes the paper.

\section{BACKGROUND}

In this section, we introduce some background knowledge for subsequent discussions. We start with a summary of XACML policy algebra [8], then present an overview of Ponder2 policy language [4], [7] and preliminary notions of policy semantics adopted in our work.

\section{A. An Algebra for XACML Policies}

Let $P$ be a 3-valued access control policy. They define the semantics of $P$ as a 2-tuple $\left\langle R_{Y}^{P}, R_{N}^{P}\right\rangle$, where $R_{Y}^{P}$ and $R_{N}^{P}$ is the set of requests that are permitted and defined by $P$ respectively. $P$ can be viewed as a function mapping each request to a value in $\{Y, N, N A\}$, determining that whether the request is allowed $(Y)$, denied $(N)$ or not-applicable $(N A)$. The Fine-grained Integration Algebra (FIA) is given by $\left\langle\Sigma, P_{Y}, P_{N},+, \&, \neg, \Pi_{d c}\right\rangle$, where $\Sigma$ is a vocabulary of attributes names and their domains, $P_{Y}$ and $P_{N}$ are two policy constants which permits and denies everything respectively, + and \& are two binary operators, and $\neg$ and $\Pi_{d c}$ are two unary operators. Operators on policies are described as set operations. The integration of policies may involve multiple operators based on the definition of FIA expressions (see [8] for a detailed description).

\section{B. An Overview of Ponder2}

The Ponder2 [7] language provides a common means of specifying security policies that map onto various access control implementation mechanisms for firewalls, operating systems, databases etc. It primairly supports two types of policies: authorization policies that defines which actions are permitted under given circumstances and obligation policies that define which actions should be performed in response to an event occurring if specific conditions are fulfilled. Ponder2 specifies policies in a subject-action-target (SAT) format, in 
additional to optional fields such as constraints, triggers etc. For example ${ }^{1}$, an authorization policy looks like the following: auth + /sensor/temperature $\rightarrow$ /pda.reportTemp()

which permits temperature sensors to perform the reportTemp action on pda. The following obligation policy specifies that the oxygen saturation sensor should be activated when the heart-rate is above 100 .

on heartrate(hr) do if $\mathrm{hr} \geq 50$ then /sensor/os.activate()

Ponder2 provides two types of authorization policies, namely positive authorization auth+ and negative authorization auth-. Only one type of obligation policy is specified in Ponder2, stating that a subject is obliged to perform certain action on that target. An obligation policy can be enforced only if the corresponding authorization policy has been specified in the system. An event field specifies the trigger of the obligation. Optional constraints may apply to both types of policies. These constraints are evaluated against the state of the system.

\section{Policy Semantics}

We propose a simple yet powerful notation of policy semantics to capture both authorization and obligation requirement to study their composition and interaction. Our representation can be easily instantiated to support a large variety of policy languages.

DEFINITION 1. A security policy is defined as an evaluation function $\mathcal{P}: \mathcal{S T} \times \mathcal{A} \rightarrow \mathcal{D}$, where $\mathcal{S} \mathcal{T}$ is the set of system states, $\mathcal{A}$ represents a finite set of actions and $\mathcal{D}$ denotes the set of decision tuples $\left\{\left\langle\mathcal{D}_{a}, \mathcal{D}_{o}\right\rangle\right\}=$ $\{\langle Y, Y\rangle,\langle Y, N A\rangle,\langle N, N A\rangle,\langle N A, N A\rangle\}$. The function $\mathcal{P}$ takes a system state $s t \in \mathcal{S T}$ and an action $a \in \mathcal{A}$ as input, and returns a decision tuple $\left\langle d_{a}, d_{o}\right\rangle$ determining whether $a$ is authorized and obliged to execute in state $s t$.

Our semantics also support three-valued policies when evaluating an authorization request. But the obligation of the execution of an action may exist $(Y)$ or may not $(N A){ }^{2}$ Note that the set of decision tuples does not include $\langle N, Y\rangle$ and $\langle N A, Y\rangle$ to ensure policy consistency since an obligation intuitively implies the corresponding positive authorization in the system. In an implementation, the system states $\mathcal{S} \mathcal{T}$ will most likely encodes concepts like principals, i.e. the entity that will execute the action, and a target, where the action will be executed and any other detail required by the execution of the action.

Definition 2. Let $\mathcal{S}$ be the set of subjects, $\mathcal{T}$ be the set of targets, $\mathcal{A}$ be the set of actions, $\mathcal{E}$ be the set of event triggers and $\mathcal{C}$ be the set of conditional constraints. We can now define system states as $\mathcal{S} \mathcal{T}=\mathcal{E} \times \mathcal{C} \times \mathcal{S} \times \mathcal{T}$. This definition allows a system state to be described as $s t=\langle e, c, s, t\rangle$ consisting of an event trigger $e \in \mathcal{E}$, the conditional constraint $c \in \mathcal{C}$, subject $s \in \mathcal{S}$ and target $t \in \mathcal{T}$ in Ponder2.

\footnotetext{
${ }^{1}$ Examples taken from [7].

${ }^{2}$ We do not consider negative obligations in our model. Because negative obligations can be easily transformed into access control requirement like refrain policies, and thus can be enforced directly.
}

Now we can easily transform a Ponder2 policy using the above definition. TABLE I illustrates some sample policies for a conference reviewing system. Policies $\left\{P_{1}, P_{2}, \ldots, P_{5}\right\}$ are written in Ponder2 language, which are transformed into $\left\{P_{1}^{\prime}, P_{2}^{\prime}, \ldots, P_{5}^{\prime}\right\}$.

\begin{tabular}{|c|c|}
\hline$P_{1}$ & $d($ () when \\
\hline$P_{2}$ & auth $+\mathrm{r}=/$ reviewer $\rightarrow \mathrm{p}=/$ paper $/$ paper.read () when $\operatorname{assign}(\mathrm{r}, \mathrm{p})=\mathrm{T}$ \\
\hline$\overline{P_{3}}$ & auth $+\mathrm{r}=/$ reviewer $\rightarrow \mathrm{p}=/$ paper $/$ paper.review () when $\operatorname{assign}(\mathrm{r}, \mathrm{p})=\mathrm{T}$ \\
\hline$P_{4}$ & auth $-\mathrm{a}=/$ author $\rightarrow \mathrm{p}=/$ paper $/$ paper.read ()$, /$ paper.review () when $\operatorname{submit}(\mathrm{a}, \mathrm{p})=\mathrm{T}$ \\
\hline$P_{5}$ & on $\operatorname{assign}(\mathrm{r}, \mathrm{p})=\mathrm{T} \mathrm{r}=/$ reviewer $\mathrm{p}=/$ paper do $/$ reviewer $\rightarrow /$ paper.review() \\
\hline$P$ & $P_{1}^{\prime}(\langle$ submit $(\mathrm{a}, \mathrm{p})=\mathrm{T}, \mathrm{a}=/$ author, $\mathrm{p}=/$ paper $\rangle, \operatorname{read}())=\langle Y, N A\rangle$ \\
\hline$\overline{P_{2}^{\prime}}$ & $P_{2}^{\prime}(\langle$ assign $(\mathrm{r}, \mathrm{p})=\mathrm{T}, \mathrm{r}=/$ reviewer, $\mathrm{p}=/$ paper $\rangle, \mathrm{read}())=\langle Y, N A\rangle$ \\
\hline$P_{3}^{\prime \prime}$ & $P_{3}^{\prime}(\langle$ assign $(\mathrm{r}, \mathrm{p})=\mathrm{T}, \mathrm{r}=/$ reviewer, $\mathrm{p}=/$ paper $\rangle$, review ()$)=\langle Y, N A\rangle$ \\
\hline $\bar{P}$ & $\bar{P}_{4}^{\prime}(\langle$ submit $(\mathrm{a}, \mathrm{p})=\mathrm{T}, \mathrm{a}=/$ author, $\mathrm{p}=/$ paper $\rangle,\{\operatorname{read}()$, review ()$\})=\langle N, N A\rangle$ \\
\hline & \\
\hline
\end{tabular}

TABLE I

SAMPLE POLICIES FOR A CONFERENCE REVIEWING SYSTEM

DEFINITION 3. Our first algebra components are the following two policy constants $\mathbf{P}_{+}: \mathcal{S T} \times \mathcal{A} \rightarrow\langle Y, N A\rangle$ and $\mathbf{P}_{-}: \mathcal{S} \mathcal{T} \times \mathcal{A} \rightarrow\langle N, N A\rangle$. More precisely, $P_{+}$specifies that every authorization request will be allowed in any state and no obligation is required in the system; whereas $\mathbf{P}_{-}$says that any authorization request is denied therefore no obligation is required.

It is quite common in a policy-managed system to adopt a default authorization policy: every action is allowed or every action is forbidden. Firewall policy is such an example. Ponder2 also allows the policy administrator to specify a default authorization policy, such as ALL+ or ALL-. Normally, default obligation requirements are not specified during system initialization. The two policy constants allow us to represent default authorization requirement in the system.

\section{An Algebra for Policies}

We are now ready to introduce the algebra extended from [8]. We first define the syntax and semantics of the basic algebraic operations and then discuss their properties and expressiveness in details.

\section{A. Basic Algebraic Operations}

The algebra consists of the two constant policies $P_{+}$and $P_{-}$ and six basic algebraic operations: addition $(+)$, intersection $(\&)$, subtraction $(-)$, projection $(\Pi)$, and two negation operations, one for authorizations $\left(\neg_{a}\right)$ and another for obligations $\left(\neg_{0}\right)$. Let $P_{1}$ and $P_{2}$ be two policies to be combined, and $P_{I}$ be the result from combination. For the ease of our discussion, we introduce three binary operators $\oplus, \otimes, \ominus$ in TABLE II. The first column of each matrix are the evaluation results of $P_{1}$ with respect to an input $(s t, a)$ and the first row are the results of $P_{2}$ with respect to the same input. Each entry denotes the effect of integrating $P_{1}$ and $P_{2}$ using $\oplus, \otimes, \ominus$ respectively. The effect of two negation operators $\neg_{a}$ and $\neg_{o}$ are also described in TABLE II.

Addition(+). Addition of $P_{1}$ and $P_{2}$ results in an integrated policy $P_{I}$ equivalent to the union of the two. 


\begin{tabular}{|c|c|c|c|c|}
\hline$P_{1}(s t, a) \oplus P_{2}(s t, a)$ & $\langle Y, Y\rangle$ & $\langle Y, N A\rangle$ & $\langle N, N A\rangle$ & $\langle N A, N A\rangle$ \\
\hline$\langle Y, Y\rangle$ & $\langle Y, Y\rangle$ & $\langle Y, Y\rangle$ & $\langle N A, N A\rangle$ & $\langle Y, Y\rangle$ \\
\hline$\langle Y, N A\rangle$ & $\langle Y, Y\rangle$ & $\langle Y, N A\rangle$ & $\langle N A, N A\rangle$ & $\langle Y, N A\rangle$ \\
\hline$\langle N, N A\rangle$ & $\langle N A, N A\rangle$ & $\langle N A, N A\rangle$ & $\langle N, N A\rangle$ & $\langle N, N A\rangle$ \\
\hline$\langle N A, N A\rangle$ & $\langle Y, Y\rangle$ & $\langle Y, N A\rangle$ & $\langle N, N A\rangle$ & $\langle N A, N A\rangle$ \\
\hline$P_{1}(s t, a) \otimes P_{2}(s t, a)$ & $\langle Y, Y\rangle$ & $\langle Y, N A\rangle$ & $\langle N, N A\rangle$ & $\langle N A, N A\rangle$ \\
\hline$\langle Y, Y\rangle$ & $\langle Y, Y\rangle$ & $\langle Y, N A\rangle$ & $\langle N A, N A\rangle$ & $\langle N A, N A\rangle$ \\
\hline$\langle Y, N A\rangle$ & $\langle Y, N A\rangle$ & $\langle Y, N A\rangle$ & $\langle N A, N A\rangle$ & $\langle N A, N A\rangle$ \\
\hline$\langle N, N A\rangle$ & $\langle N A, N A\rangle$ & $\langle N A, N A\rangle$ & $\langle N, N A\rangle$ & $\langle N A, N A\rangle$ \\
\hline$\langle N A, N A\rangle$ & $\langle N A, N A\rangle$ & $\langle N A, N A\rangle$ & $\langle N A, N A\rangle$ & $\langle N A, N A\rangle$ \\
\hline$P_{1}(s t, a) \ominus P_{2}(s t, a)$ & $\langle Y, Y\rangle$ & $\langle Y, N A\rangle$ & $\langle N, N A\rangle$ & $\langle N A, N A\rangle$ \\
\hline$\langle Y, Y\rangle$ & $\langle N A, N A\rangle$ & $\langle N A, N A\rangle$ & $\langle N A, N A\rangle$ & $\langle Y, Y\rangle$ \\
\hline$\langle Y, N A\rangle$ & $\langle N A, N A\rangle$ & $\langle N A, N A\rangle$ & $\langle N A, N A\rangle$ & $\langle Y, N A\rangle$ \\
\hline$\langle N, N A\rangle$ & $\langle N A, N A\rangle$ & $\langle N A, N A\rangle$ & $\langle N A, N A\rangle$ & $\langle N, N A\rangle$ \\
\hline$\langle N A, N A\rangle$ & $\langle N A, N A\rangle$ & $\langle N A, N A\rangle$ & $\langle N A, N A\rangle$ & $\langle N A, N A\rangle$ \\
\hline$P(s t, a)$ & $\langle Y, Y\rangle$ & $\langle Y, N A\rangle$ & $\langle N, N A\rangle$ & $\langle N A, N A\rangle$ \\
\hline$\neg_{a} P(s t, a)$ & $\langle N, N A\rangle$ & $\langle N, N A\rangle$ & $\langle Y, N A\rangle$ & $\langle N A, N A\rangle$ \\
\hline$\neg_{o} P(s t, a)$ & $\langle Y, N A\rangle$ & $\langle Y, Y\rangle$ & $\langle N, N A\rangle$ & $\langle N A, N A\rangle$ \\
\hline
\end{tabular}

TABLE II

POLICY COMBINATION MATRIX FOR OPERATORS $\oplus, \otimes, \ominus, \neg a, \neg o$

$$
\begin{aligned}
P_{I} & =P_{1}+P_{2}, \text { such that } \\
P_{I}(s t, a) & =P_{1}+P_{2}(s t, a) \\
& =P_{1}(s t, a) \oplus P_{2}(s t, a)
\end{aligned}
$$

Intersection(\&). Intersection of $P_{1}$ and $P_{2}$ results in policy $P_{I}$ equivalent to the conjunction of the two.

$$
\begin{aligned}
P_{I} & =P_{1} \& P_{2}, \text { such that } \\
P_{I}(s t, a) & =P_{1} \& P_{2}(s t, a) \\
& =P_{1}(s t, a) \otimes P_{2}(s t, a)
\end{aligned}
$$

Negation $\left(\neg_{a}, \neg_{o}\right)$. Negation of a policy $P$ returns $P_{I}$ which permits (denies) all requests denied (permitted) by $P$. The operator $\neg a$ is used to negate the result of evaluating an authorization request. It does not modify the obligation part with the only exception that $\neg_{a} P_{I}(s t, a)=\langle N, N A\rangle$ if $P(s t, a)=\langle Y, Y\rangle$ (since $\langle N, Y\rangle$ is not valid). Similarly, the operator $\neg_{o}$ is used to negate the evaluation result of an obligation request without touching the authorization part.

$$
P_{I}=\neg P \text {, such that }
$$$$
P_{I}(s t, a)=\neg P(s t, a) \text {, where } \neg \in\left\{\neg a, \neg_{o}\right\}
$$

Subtraction(-). Subtracting policy $P_{2}$ from $P_{1}$ results in the policy $P_{I}$ which applies only to those requests that $P_{2}$ does not apply to. Also the subtraction operation can be expressed in terms of $\left\{+, \&, \neg_{a}, \neg_{o}\right\}$.

$$
\begin{aligned}
P_{I} & =P_{1}-P_{2}, \text { such that } \\
P_{I}(s t, a) & =P_{1}-P_{2}(s t, a) \\
& =P_{1}(s t, a) \ominus P_{2}(s t, a) \\
& =\left(P_{1}+\neg_{a} P_{2}\right) \&\left(P_{1}+\neg_{o} P_{2}\right)(s t, a)
\end{aligned}
$$

Projection( $(\Pi)$. The projection operation is used to extract a portion of a given policy $P$. Let $c(\mathcal{S T} \times \mathcal{A})$ be a computable subset of $\mathcal{S T} \times \mathcal{A}$. The projection operation restricts the policy $P$ based on the specified evaluation result of request $(s t, a) \in c(\mathcal{S} \mathcal{T} \times \mathcal{A})$. The usper-index $\left\langle d_{a}, d_{o}\right\rangle$ is optional. If it does not appear, the second condition in the "if" is ignored.

$$
\begin{aligned}
& P_{I}=\Pi_{c(\mathcal{S} \mathcal{T} \times \mathcal{A})}^{\left\langle d_{a}, d_{o}\right\rangle} P \text {, such that } \\
& P_{I}(s t, a)=\Pi_{c(\mathcal{S} \mathcal{T} \times \mathcal{A})}^{\left\langle d_{a}, d_{o}\right\rangle} P(s t, a) \\
& = \begin{cases}\left\langle d_{a}, d_{o}\right\rangle & \text { if }(s t, a) \in c(\mathcal{S T} \times \mathcal{A}) \\
\langle N A, N A\rangle & \text { and } P(s t, a)=\left\langle d_{a}, d_{o}\right\rangle \\
\langle\text { otherwise }\end{cases}
\end{aligned}
$$

So far we have defined the policy algebra consisting of two policy constants and six basic operations. Note that the integration of policies may involve a sequence of operations as discussed in [8].

\section{B. Properties and Expressiveness of the Algebra}

The policy algebra has all the algebraic properties listed in [8] except for those involving negation operations. Instead, we have the following properties:

1) $\neg_{a}\left(P_{1}+P_{2}\right)=\neg_{a} P_{1}+\neg_{a} P_{2}$;

2) $\neg_{a}\left(P_{1} \& P_{2}\right)=\neg_{a} P_{1} \& \neg_{a} P_{2}$;

3) $P_{+}=\neg_{a} P_{-}, P_{-}=\neg_{a} P_{+}$;

4) $\neg_{o} \neg_{o} P=P$.

In section II, we introduced two constant policies $P_{+}$and $P_{-}$. The other two constant policies $P_{Y}: \mathcal{S T} \times \mathcal{A} \rightarrow\langle Y, Y\rangle$ and $P_{N A}: \mathcal{S T} \times \mathcal{A} \rightarrow\langle N A, N A\rangle$ can be produced using $P_{+}$ and $P_{-}$. For example, $P_{Y}=\neg_{0} P_{+}$and $P_{N A}=P_{+}+P_{-}=$ $P_{+} \& P_{-}$.

COROLlary of THEOREM 3 in [8]. Given any policy integration matrix $M$, let $M\left(P_{1}, P_{2}\right)$ be the result of integrating policies $P_{1}$ and $P_{2}$ using matrix $M$. We can always find an algebra expression $\mathcal{E}$ such that $\mathcal{E}\left(P_{1}, P_{2}\right)=M\left(P_{1}, P_{2}\right)$, where $\mathcal{E}$ consists of $\left\{P_{+}, P_{-},+,-, \&, \neg_{a}, \neg_{o}, \Pi\right\}$. Therefore, our algebra is complete and the detailed proof is removed for space conservation.

\section{Policy Integration And Analysis}

In this section, we describe mechanisms for policy integration and analysis using algebraic operations defined previously.

\section{A. Policy Integration and Analysis}

The integration of policies $P_{1}, P_{2}, \ldots, P_{n}$ can be easily expressed as $P_{I}=P_{1}+P_{2}+\ldots+P_{n}$. The result of policy integration $P_{I}$ permits requests that are permitted by at least one of the policies; It denies requests that are denied by at least one of them. If a request is permitted by one policy but denied by another policy, $P_{I}$ returns $\langle N A, N A\rangle$ and does not make decision on the request. Sometimes, we may also want to extract desired parts of individual policies and then combine them into an integrated one. We can, for example, take the allows from $P_{1}$ and the denies from $P_{2}$ using $P_{I}=\Pi^{\left\langle Y, d_{o_{1}}\right\rangle} P_{1}+\Pi^{\left\langle N, d_{o_{2}}\right\rangle} P_{2}$. As we discussed in SECTION II, it is quite common in a policy-managed system to adopt a default authorization policy: every action is allowed $\left(P_{+}\right)$ or every action is forbidden $\left(P_{-}\right)$. Therefore, any request that is evaluated to $\langle N A, N A\rangle$ by the integrated policy $P_{I}$ will take the result specified by the default policy $P_{d}$. Thus we have $P_{I}^{\prime}=P_{I}+\left(P_{\text {def }}-P_{I}\right)$, where $P_{\text {def }} \in\left\{P_{+}, P_{-}, \neg_{o} P_{+}\right\}$ depending on the system initialization.

It has been long recognized that merely providing a policy editing tool to ensure correct policy syntax is not sufficient. Policies can interact with each other, often with undesirable effects as pointed by [2]. With the support of policy algebra, we can perform policy analysis upon enforcement. 
1) Dominance Check: Dominance check is important since any ineffective policy must not be deployed so that CPU cycles are not wasted on evaluating it. We say that a policy $P$ is dominated by the already existing policy $P^{\prime}$ if $P$ is ineffective when added into the policy set because of the existence of policy $P^{\prime}$, i.e. there is no resource in the domain whose operation will not be affected by $P$. For example, a policy that permit the creation of usernames only "username $\geq 4$ " is dominated by another policy for which " $4 \leq$ username $\leq 10$ ". Given two policies $P$ and $P^{\prime}$, we say that $P$ is dominated by $P^{\prime}$ if and only if $P+P^{\prime}=P^{\prime}$ or $P \& P^{\prime}=P$. This definition also applies to two sets of policies. Given $P_{I}=P_{1}+P_{2}+\ldots+P_{m}$ and $P_{I}^{\prime}=P_{1}^{\prime}+P_{2}^{\prime}+\ldots+P_{n}^{\prime}$, we say that $P_{I}$ is dominated by $P_{I}^{\prime}$ if and only if $P_{I}+P_{I}^{\prime}=P_{I}^{\prime}$ or $P_{I} \& P_{I}^{\prime}=P_{I}$.

2) Coverage Check: When specifying policies for management systems, the administrator may want to know if explicit policies have been defined for a certain range of input parameters. The input parameters of our interests would be certain set of system states $\mathcal{S} \mathcal{T}$, and action set $\mathcal{A}$. That is, given a computable subset $c(\mathcal{S T} \times \mathcal{A})$ of $\mathcal{S T} \times \mathcal{A}$, we want to make sure that $\prod_{c(\mathcal{S} \mathcal{T} \times \mathcal{A})} P(s t, a) \neq\langle N A, N A\rangle$ for all input requests $(s t, a) \in c(\mathcal{S} \mathcal{T} \times \mathcal{A})$. Similarly, coverage check can also be performed upon a set of policies.

3) Conflict Detection and Resolution: Policy conflicts can arise due to omissions, errors or conflicting requirement of the administrators specifying the policies. For example, there may be two authorization policies which permit and forbid the same activity; or an obligation policy may define an activity which is forbidden by a negative authorization policy. We say that two policies are in conflict if they cannot be satisfied simultaneously [6]. Given two policies $P_{1}$ and $P_{2}$, we know that they conflict with each other if and only if $P_{1}(s t, a) \neq$ $P_{2}(s t, a)$ for some $(s t, a) \in \mathcal{S T} \times \mathcal{A}$ The key point for conflict resolution is to assign proper precedence between policies. There are three possibilities:

- $P_{I_{1}}=P_{1}+\left(P_{2}-P_{1}\right)$ : gives higher precedence to $P_{1}$. That is, $P_{I}(s t, a)=P_{1}(s t, a)$ for all (st, a) such that $P_{1}(s t, a) \neq P_{2}(s t, a)$.

- $P_{I_{2}}=P_{2}+\left(P_{1}-P_{2}\right)$ : gives higher precedence to $P_{2}$. Similarly, $P_{I}(s t, a)=P_{2}(s t, a)$ for all (st, a) such that $P_{1}(s t, a) \neq P_{2}(s t, a)$.

- $P_{I_{d}}=\left(P_{1}+P_{2}\right)+\left(P_{d e f}-\left(P_{1}+P_{2}\right)\right)$ solves the conflicts by applying the default policy to requests that receive conflicting results from $P_{1}$ and $P_{2}$.

\section{B. Examples in Ponder2}

In this subsection, we provide some concrete examples of Ponder2 policy analysis. TABLE I lists a few sample policies $\left\{P_{1}, P_{2}, \ldots, P_{5}\right\}$ written in Ponder2 language. They are transformed into $\left\{P_{1}^{\prime}, P_{2}^{\prime}, \ldots, P_{5}^{\prime}\right\}$ using the proposed policy semantics.

EXAMPLE 1: As an example of policy combination, we can perform $P_{I}=P_{1}^{\prime}+P_{2}^{\prime}$ to obtain the integrated policy $P_{I}$. More precisely, for a request $(s t, a)$, we have $P_{I}(s t, a)=\langle Y, N A\rangle$ if and only if $s t \in\{\langle\operatorname{submit}(\mathrm{a}, \mathrm{p})=$ true, $\mathrm{a}=/$ author, $\mathrm{p}=/$ paper $\rangle$, $\langle\operatorname{assign}(\mathrm{r}, \mathrm{p})=\mathrm{T}, \mathrm{r}=/$ reviewer, $\mathrm{p}=/$ paper $\rangle\}$ and $a=\operatorname{read}() ;$ Otherwise, $P_{I}(s t, a)=\langle N A, N A\rangle$. That is, the integrated policy $P_{I}$ allows both the author and the assigned reviewer to read the paper. Similarly, we could perform $P_{I}^{\prime}=P_{2}^{\prime}+P_{3}^{\prime}$ and the resulting policy $P_{I}^{\prime}$ allows the reviewer to read and review the assigned paper.

EXAMPLE 2: By combining $P_{3}^{\prime}$ and $P_{5}^{\prime}$, we observe that $P_{I}^{\prime}=P_{3}^{\prime}+P_{5}^{\prime}=P_{5}^{\prime}$. This is because in the proposed policy semantics, the enforcement of an obligation policy implies that the corresponding authorization requirement must be specified to ensure consistency.

EXAMPLE 3: We notice that $P_{1}^{\prime}$ and $P_{4}^{\prime}$ conflict with each other. Because $P_{1}^{\prime}(s t, a)=\langle Y, N A\rangle$ but $P_{4}^{\prime}(s t, a)=\langle N, N A\rangle$ when $s t \in\{\langle\operatorname{submit}(\mathrm{a}, \mathrm{p})=\mathrm{T}, \mathrm{a}=/$ author, $\mathrm{p}=/$ paper $\rangle\}$ and $a=$ $\operatorname{read}()$. We can solve this conflict by giving precedence to $P_{1}^{\prime}$. That is, $P_{I}^{\prime}=P_{1}^{\prime}+\left(P_{2}^{\prime}-P_{1}^{\prime}\right)$, such that $P_{I}^{\prime}($ st , read ()$)=$ $\langle Y, N A\rangle$ and $P_{I}^{\prime}($ st, review ()$)=\langle N, N A\rangle$. Thus, we allow the author to read his own paper but he cannot review it. In reality, it is the system administrator's decision to assign precedence among conflicting policies.

\section{CONClusion}

To address the importance of obligation policies, and study the interaction between authorization and obligation requirements, we propose an algebra for Ponder 2 policies, and provide mechanisms for policy integration and analysis using the algebraic operations.For future work, we would like to accomplish the algebra implementation using Ponder2 language. We also plan to study the interaction between access control policies and obligation policies in great depth. For example, the current version of the algebra is not able to handle obligations that may depend on more than one system state. We would like to improve it in the future work.

\section{REFERENCES}

[1] "Extensible access control makeup language (xacml) version 2.0," OASIS Standard, 2005.

[2] D. Agrawal, J. Giles, K. won Lee, and J. Lobo, "Policy ratification," in Proceedings of IEEE Policy 2005, 2005.

[3] P. Bonatti, S. D. C. di Vimercati, and P. Samarati, "An algebra for composing access control policies," ACM Transactions on Information and System Security (TISSEC), vol. 5, no. 1, pp. 1-35, Feb. 2002.

[4] N. Damianou, N. Dulay, E. Lupu, and M. Sloman, "The ponder policy specification language," in Proceedings of IEEE Policy 2001, 2001.

[5] K. Irwin, T. Yu, and W. H. Winsborough, "On the modeling and analysis of obligations," in ACM Conference on Computer and Communications Security (CCS), 2006.

[6] E. C. Lupu and M. Sloman, "Conflicts in policy-based distributed systems management," in IEEE Transactions on Software Engineering, vol. 25 , no. 6 , 1999 , pp. 852-869.

[7] E. Lupu, N. Dulay, A. S. Filho, S. Keoh, M. Sloman, and K. Twidle, "Amuse: Autonomic management of ubiquitous e-health systems," in Concurrency and Computation: Practice and Experience, wiley, 2007.

[8] P. Rao, D. Lin, E. Bertino, N. Li, and J. Lobo, "An algebra for finegrained integration of xacml policies," CERIAS Tech Report 2001-124, Purdue University, 2007.

[9] D. Wijesekera and S. Jajodia, "A propositional policy algebra for access control," ACM Transactions on Information and System Security (TISSEC), vol. 6, no. 2, pp. 286-325, May 2003.

[10] H. Zhao and S. M. Bellovin, "Policy algebras for hybrid firewalls," in Annual Conference of ITA (ACITA) 2007, 2007. 